\title{
Relativistic effects on imaging by a rotating optical system ${ }^{\star}$
}

\author{
G. Anglada-Escudé ${ }^{1}$, S. A. Klioner ${ }^{2}$, M. Soffel ${ }^{2}$, and J. Torra ${ }^{1}$ \\ 1 Departament d'Astronomia i Meteorologia, Universitat de Barcelona, Av. Diagonal 647, 08028 Barcelona, Spain \\ 2 Lohrmann Observatory, Dresden Technical University, Mommsenstr. 13, 01062 Dresden, Germany
}

Received 7 October 2005 / Accepted 29 September 2006

\section{ABSTRACT}

\begin{abstract}
Context. High accuracy astrometric instruments like Gaia aiming at an accuracy of 1 microarcsecond cannot be considered as pointlike observers in the framework of relativistic modelling of observable quantities.

Aims. Special-relativistic effects on the imaging by a non-point-like arbitrarily moving optical instrument are discussed.

Methods. A special-relativistic reflection law for a mirror of arbitrary shape and motion is derived in the limit of geometrical optics. The aberration patterns are computed with ray tracing using a full special-relativistic model for two simple rotating optical instruments.

Results. The effect of special-relativistic reflection law on the photocenters of the aberration patterns of an optical system rotating with a moderate angular velocity of $60^{\prime \prime} / \mathrm{s}$ may be at the level of 1 microarcsecond if the system involves mirrors significantly inclined relative to the optical axis.

Conclusions. Special-relativistic optical modelling of future astrometric instruments is indispensable if a level of a few microarcseconds is envisaged.
\end{abstract}

Key words. astrometry - reference systems - relativity

\section{Introduction}

We investigate possible relativistic effects on the imaging of an optical system with arbitrary motion. In the framework of relativity one usually considers point-like observers. The methods to calculate observed quantities for such observers are well known. It is common to assume that the actual instrumentation of the observer is so small that one considers the positions and velocities of each part of the instrument to be the same (and that single position and velocity is called the position and velocity of the observer). In reality even for an Earth-based telescope the velocities of different parts of the primary mirror in inertial coordinates (not rotating with the Earth) are slightly different. However, in the past the accuracy of observations was considered to be "too low" and the size of the mirror "too small" for those differences to be of practical relevance.

Due to recent technical developments especially for astrometric space missions like Gaia (de Boer et al. 2000; Perryman et al. 2001; Bienaymé \& Turon 2002), JASMINE (Gouda et al. 2002) and SIM (Shao 1998) the situation has changed. In the case of Gaia, we deal with a scanning satellite which permanently rotates in space with a period of $6 \mathrm{~h}$. The size of the primary mirror of Gaia is $1.4 \mathrm{~m}$, comparable with the size of the spacecraft itself. The envisaged best accuracy of Gaia is a few $\mu$ as (and can be even below that limit in some favorable cases). Therefore, one cannot neglect a priori the difference of velocities of various parts of the instruments. It is our purpose to investigate these effects and estimate their magnitude for Gaia.

The general-relativistic model for Gaia has been formulated by Klioner $(2003,2004)$. The model uses two principal relativistic reference systems: (1) the Barycentric Celestial Reference System (BCRS) and (2) the Center of Mass Reference System

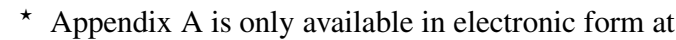
http: //www . aanda. org
(CoMRS) of the satellite. The former is a global reference system with its origin at the barycenter of the solar system. It has been recommended by the International Astronomical Union for relativistic modelling of high-accuracy astronomical observations (Soffel et al. 2003). This reference system is used to model the dynamics of massive bodies, space vehicles (e.g., the Gaia satellite) and light rays within the Solar system. The final Gaia catalogue will contain coordinates of celestial objects in the BCRS. The CoMRS is the local relativistic reference system of the satellite. The theory of such local reference systems was laid down by Ni \& Zimmermann (1978) and then elaborated by Klioner \& Voinov (1993) and Klioner (2004). The gravitational influence of massive bodies is reduced in the CoMRS as much as possible and, according to the equivalence principle, is represented by tidal potentials. The CoMRS has its origin in the center of mass of the satellite and is kinematically non-rotating with respect to the BCRS. The CoMRS is physically adequate to model phenomena occurring in the immediate neighborhood of the satellite: attitude, the process of observation, etc. According to Klioner (2004) the metric tensor of the CoMRS differs from the Minkowski metric in three kinds of terms (the gravitational field of the satellite is too small and can be neglected safely): an inertial term due to non-gravitational accelerations of the satellite (for Gaia these accelerations can be relatively large during orbital maneuvers and only about $2 \times 10^{-13} \mathrm{~m} / \mathrm{s}^{2}$ in between, mainly due to solar pressure); an inertial term due to the slow rotation of the CoMRS relative to the co-moving Fermi-Walker transported locally inertial reference system (with an angular velocity of $\sim 3 \times 10^{-15} \mathrm{~s}^{-1}=2^{\prime \prime}$ per century); and tidal gravitational potentials (producing relative accelerations of at most $10^{-12} \mathrm{~m} / \mathrm{s}^{2}$ at a distance of $2.5 \mathrm{~m}$ from the satellite's center of mass). Simple calculations show that all these terms influence the CoMRS light propagation within a few meters from the satellite's center of mass at a level much lower than the goal 
accuracy of $1 \mu$ as. Therefore, all these terms can be neglected for our purposes and one can consider the CoMRS for a sufficiently small interval of time as an inertial reference system of Special Relativity.

In Sect. 2 we summarize how to calculate the specialrelativistic effects in the aberration patterns due to the rotation of the instrument. Section 3 is devoted to a description of ray tracing calculations of the relativistic effects in the aberration patterns for two simple optical systems. The details of the derivation of the special-relativistic deflection law are given in the appendix. There we also introduce a general theoretical scheme we use to treat arbitrarily-shaped and arbitrarily moving mirrors in special relativity.

\section{General scheme of computing relativistic effects due to the rotation of an optical system}

Our goal is to discuss and calculate the influence of relativistic effects on the imaging by an optical instrument with some noninertial motion. We simplify our goal in several directions: (1) we consider here the case of optical instruments consisting of mirrors only (no lenses are considered), (2) we do not consider the effects of wave optics and work in the approximation of geometric optics (see, however, the note at the end of Sect. 4).

For an optical system consisting solely of a number of arbitrarily moving mirrors, the most important relativistic effect is the special-relativistic modification of the reflection law. That modified special-relativistic reflection law will produce a change in aberration patterns as compared to the patterns calculated by using the usual reflection law (here and below by "usual reflection law" we mean that the angles between the normal to the surface of the mirror and the incoming and reflected light ray are equal: $\alpha^{\prime}=\alpha$ in Fig. A.1). These perturbed aberration patterns could affect astrometric measurements based on an interpretation of the images obtained in the instrument's focal plane.

\subsection{Reflection law}

First, we formulate the general principles allowing one to calculate the aberration patterns within the framework of Special Relativity. Given a mirror of arbitrary shape in arbitrary motion (see Sect. A.2 for a formal mathematical description of such an arbitrary mirror and Sect. 2.2 for a discussion of such mirrors from the physical point of view) and a light ray hitting the surface of the mirror at a given point and moment of time, we calculate the parameters of the outgoing (reflected) light ray. The simplified problem of a flat mirror moving with a constant velocity perpendicular to its surface has been considered by Einstein (1905) in the first paper on Special Relativity Theory. In the appendix the most general case of this problem within Special Relativity is considered in great detail. Slightly modifying the arguments of Einstein (1905) we first use Lorentz transformations to transform from a laboratory inertial reference system $\left(t, x^{i}\right)$ to an inertial reference system $\left(T, X^{a}\right)$ instantaneously comoving with the element of the mirror where the reflection of a particular light ray occurs, then apply the known reflection law in that reference system and transform the reflected light ray back into the laboratory reference system. The relation of that scheme to direct calculations involving Maxwell's equations is also discussed in the appendix. In our calculations we recover a number of known results for various particular cases. An overview of these known results and the corresponding comparison are also given. The main formula used in all the ray tracing calculations of Sect. 3 is the relativistic reflection law given by Eq. (A.44).

\subsection{Arbitrarily shaped and moving mirrors}

A very important point of the whole scheme is that the shapes of the mirrors in laboratory coordinates $\left(t, x^{i}\right)$ and, possibly, the time-dependence of these shapes are assumed to be given. We describe the shape of each mirror by a two-parameter family of worldlines of each individual particle of the mirror denoted as $x_{m}^{i}(t ; \xi, \eta)$. Here $\xi$ and $\eta$ are two continuous parameters "numbering" the particles that constitute the surface of the mirror. Clearly, for fixed values of $\xi$ and $\eta$, function $x_{m}^{i}(t ; \xi, \eta)$ represents the $\left(t, x^{i}\right)$-parametrization of the world line of the corresponding particle. For fixed $t$ the same function $x_{m}^{i}(t ; \xi, \eta)$ represents the instantaneous position and shape of the mirror in the $t=$ const. hyperplane of the coordinates $\left(t, x^{i}\right)$. In this case ( $t=$ const.) the parameters $\xi$ and $\eta$ give a non-degenerated twodimensional coordinate chart on the surface of the mirror. We consider $x_{m}^{i}(t ; \xi, \eta)$ to be differentiable with respect to $\xi$ and $\eta$. This means that the coordinate representation of the surface is a smooth two-dimensional surface for each moment of coordinate time $t$.

In general there is no inertial reference system where the whole system or any of its mirrors is at rest. In the special cases when such an inertial rest-frame of a mirror does exist, one should consider the shape of the mirror in that rest-frame. In the practical cases considered below such rest-frames do not exist. Moreover, the size of the mirrors is so large that we cannot assume that the velocities of all points of the mirror are approximately constant in any inertial reference system.

We do not consider the question of deformations of the mirrors due to their non-inertial (for example, rotational) motion (i.e., the relation between the intended shapes of the mirrors during their manufacturing and their shapes, e.g., in a rotating satellite, in coordinates $\left.\left(t, x^{i}\right)\right)$. The behaviour of a mirror as a physical body is a separate question, a rigorous relativistic treatment of which would require at least a special-relativistic theory of elasticity. As long as the angular velocity is constant the deformations and special-relativistic effects on the shape (e.g. Lorentz contraction) are also constant. In this case a rigidly rotating mirror can be considered to be Born-rigid (Pauli 1958, Sect. 45). We can also argue that the constant deformations are assumed to be properly taken into account during manufacturing so that the rotating mirrors have the assumed forms. One may argue that the mirrors could be made active to retain the prescribed form (which is the case for many larger Earth-bound instruments, but may appear to be a rather bizarre argument in some other cases).

\subsection{Observable aberration patterns}

The last issue is the definition of the observing (imaging) device. In analogy to our representation of the mirrors we first define a coordinate "plane" $x_{f}^{i}(t ; \zeta, \chi)$ in laboratory coordinates $\left(t, x^{i}\right)$ that coincides with the focal "plane" of the instrument in the Newtonian case. In many cases (e.g. for the case considered in Sect. 3 below) $x_{f}^{i}(t ; \zeta, \chi)$ can be taken to be a plane in the considered coordinates (that is, for any moment of time there exist $n^{i}(t)$ independent of $\zeta$ and $\chi$ such that $\boldsymbol{x}_{f} \cdot \boldsymbol{n}=0$ ). The aberration patterns we calculate below are defined as the set of points at which the light rays from a source hit that coordinate focal plane at some moment $t=t_{\mathrm{obs}}=$ const. Generally speaking the aberration patterns cannot be considered as "infinitely small". This 
means that there is no inertial coordinate system in which the part of the detector (that is, of the focal "plane") registering an aberration pattern can be considered at rest.

If the patterns are "small enough" (which is the typical case for reasonable high-quality optical instruments) one could introduce an inertial reference system $\left(\tau, \rho^{i}\right)$ instantaneously comoving with some central point of the aberration pattern and define the "observable" pattern as a set of points at which the light rays from a source hit that coordinate focal plane at some moment $\tau=\tau_{\mathrm{obs}}=$ const. (here one should also take into account the relativistic effects in spatial coordinates and correspondingly treat Lorentz contraction etc.). First, although this approach seems to be more adequate for non-inertial motion it still gives a coordinate-dependent picture because of finite extension of the patterns. Second, we have explicitly checked that this additional Lorentz boost does not influence any of the figures and numerical results given below.

Note that we are interesting in prediction of the changes in the aberration patterns compared to the prediction made for the "same" optical device without rotation and using Newtonian geometric optics (this latter prediction is typically available from the manufacturers of the instrumentation). From this point of view, our definition of "observed" aberration pattern is adequate. In more realistic case one has to model the process of observation in much more detail (e.g., CCD orientation and position within the instrument, CCD clocking, averaging, TDI mode etc.). Such a detailed modelling is however unnecessary for the purposes of this paper.

Summarizing, our aberration pattern modelling consists of (1) fixing the models of the mirrors $x_{m}^{i}(t ; \xi, \eta)$ and the focal plane $x_{f}^{i}(t ; \zeta, \chi)$, and (2) tracing a grid of incoming light rays, which interact with the optical system only at the moments of reflection according to (A.44), until the point of intersection with the focal plane $x_{f}^{i}(t ; \zeta, \chi)$, and (3) forming the aberration pattern itself and/or calculating its photocenter.

\section{Relativistic astrometric effects due to rotational motion of the satellite}

In order to evaluate the relativistic effects in the aberration patterns of planned scanning astrometric instruments, we consider an extended optical system rotating rigidly with a constant angular velocity relative to the inertial reference system $\left(t, x^{i}\right)$. For a scanning astrometric satellite the real angular velocity is not constant (e.g., because of the required scanning law), but its changes are small and slow, and will be neglected here. Rigid rotation of the optical instrument means that the whole instrument is at rest in a reference system $\left(t, y^{i}\right)$ related to the inertial laboratory reference system $\left(t, x^{i}\right)$ as $y^{i}=R^{i}{ }_{j} x^{j}, R^{i}{ }_{j}$ being an orthogonal (rotational) matrix.

To calculate the aberration patterns of several optical systems discussed below we have developed a numerical ray tracing code in Java allowing us to calculate aberration patterns for an arbitrary optical system rigidly rotating in our laboratory coordinates. Each mirror in the system can be individually shaped and oriented in those coordinates. The code allows us to control all intermediate calculations as well as the overall numerical accuracy.

Parameters of the optical systems (size of the mirrors, focal distance, distance of the primary mirror from the rotational axis and angular velocity) considered in Sects. 3.1 and 3.2 below are chosen to qualitatively represent some principal features of planned astrometric missions like Gaia (Perryman et al. 2001) or

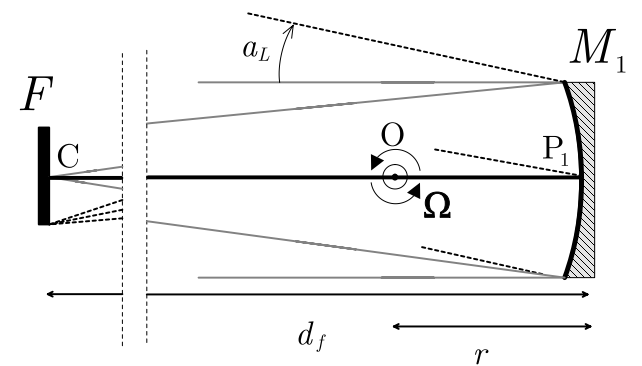

Fig. 1. A rotating optical system with one mirror.

JASMINE (Gouda et al. 2002), where a scanning satellite comprising two astrometric telescopes continuously rotates with an angular velocity of $\Omega \sim 60^{\prime \prime} / \mathrm{s}$.

\subsection{A one-mirror optical system}

The first optical system that we will study consists of one rotating parabolic mirror. A diagram of this optical system is given on Fig. 1. The parabolic mirror $M_{1}$ is a square mirror of size $1.5 \mathrm{~m} \times 1.5 \mathrm{~m}$ and a focal distance of $d_{\mathrm{f}}=46.67 \mathrm{~m}$. The receiver at the focal plane is considered to be $0.814 \mathrm{~m} \times 0.814 \mathrm{~m}$ in size providing a field of view of $\sim 1^{\circ} \times 1^{\circ}$. This roughly corresponds to the astrometric instruments of Gaia. The rotational axis goes through the origin $\mathrm{O}$ of our coordinates perpendicular to the plane of Fig. 1. The distance from $\mathrm{O}$ to the center of the primary mirror (being the vertex of the parabola) $\mathrm{P}_{1}$ is $r=1.5 \mathrm{~m}$. The distance from $\mathrm{P}_{1}$ to the center of the focal plane $\mathrm{C}$ is obviously the focal distance $d_{\mathrm{f}}=46.67 \mathrm{~m}$. The whole optical system is rotating with respect to $\mathrm{O}$ with an angular velocity $\Omega=60^{\prime \prime} / \mathrm{s}$. The optical axis of the system is defined as the path of the light ray which goes perpendicular to the surface of the primary mirror through its center provided that the system does not rotate (represented in Fig. 1 by the bold horizontal line going from $\mathrm{P}_{1}$ to $C$ ). Without rotation light rays parallel to the optical axis converge to the single point $C$ in the focal plane. The direction of an incoming light ray is parameterized with two angles: the along scan angle $a_{\mathrm{L}}$ (this angle is changing continuously for a given source because of the rotation; see Fig. 1) and the across scan angle $a_{\mathrm{C}}$. The along scan angle is the angle between the instantaneous directions of the optical axis and the incoming light ray projected into the plane containing the optical axis and perpendicular to the vector of angular velocity of the system (i.e., the plane of Fig. 1). The across scan angle is the angle between the instantaneous directions of the optical axis and the incoming light ray projected into the plane containing both the optical axis and the vector of angular velocity. The along scan and across scan angles are widely used in the context of scanning astrometric missions like HIPPARCOS (Perryman et al. 1997) and Gaia (Perryman et al. 2001).

In order to evaluate the effects due to the rotation of the instrument we calculate aberration patterns for different values of the field angles $a_{\mathrm{L}}$ and $a_{\mathrm{C}}$ as well as the differences of the photocenters for each considered case. To compute aberration patterns a rectangular grid of parallel incoming light rays with direction characterized by some given $a_{\mathrm{L}}$ and $a_{\mathrm{C}}$ is generated. These light rays are then traced through the optical system until they intersect the focal plane. The coordinates of the intersection points produce the corresponding aberration pattern in the focal plane (see, e.g., Figs. 2 and 4). The photocenter of a pattern is defined as the mean position of all points of that pattern. 

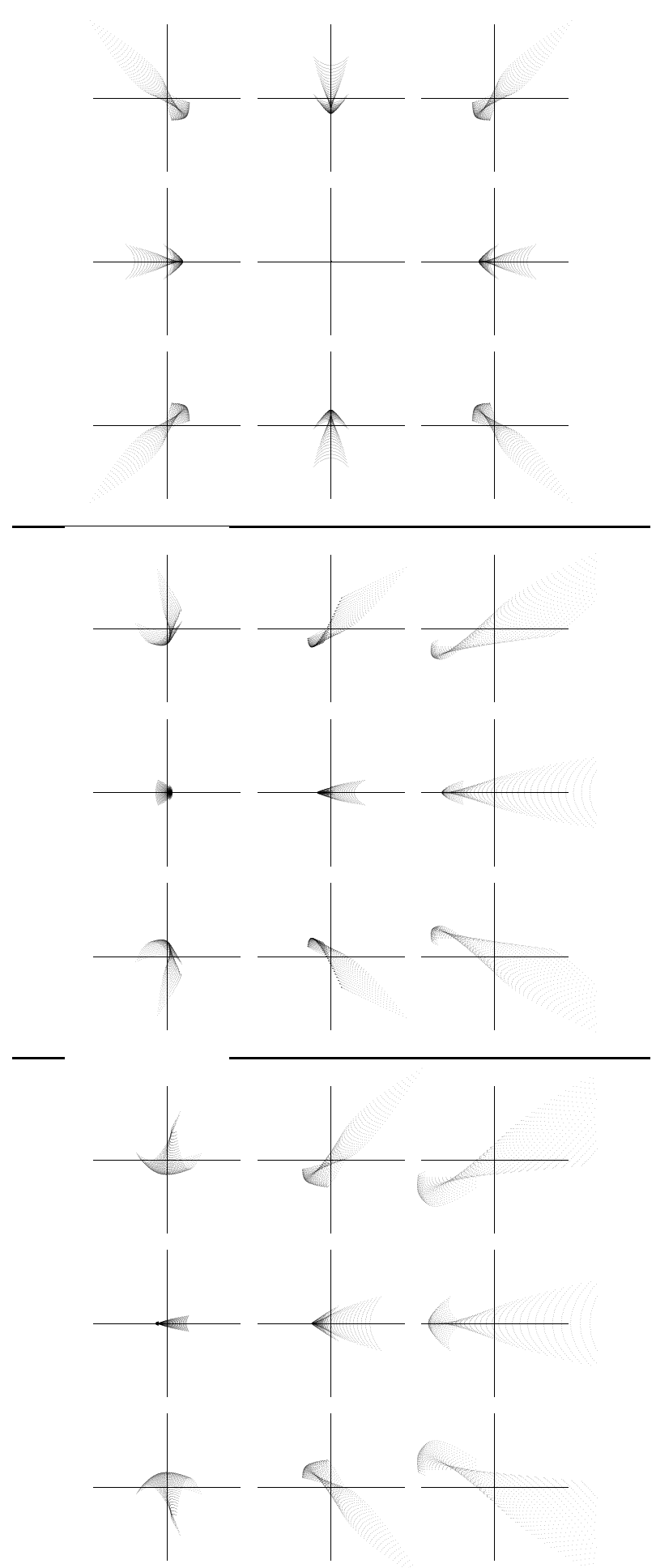

Fig. 2. Aberration patterns for the one-mirror system: a non-rotating instrument (upper panel), a rotating instrument considering the light propagation delays and using the usual reflection law (middle panel, and a rotating instrument considering both the light propagation delays and the relativistic reflection law (lower panel).

We distinguish between two different effects changing the aberration patterns (and their photocenters) of a rotating instrument compared to those of an identical non-rotating instrument. The first effect is the change of orientation of various reflecting surfaces during the time delays needed for a light ray to propagate from the primary mirror to the focal plane. The second effect is the difference between the usual reflection law and the relativistic one.

Clearly, the propagation delays are related only to the finiteness of the light velocity. The delays appear also in the nonrotating case, but can be completely ignored since the orientation of all reflecting surfaces is constant. For a rotating instrument the propagation delays mean, in particular, that the light rays producing an aberration pattern (that is, the light rays intersecting the focal plane at the same moment of time) hit the primary mirror (and, generally speaking, all other mirrors) at different times. The effect of propagation delays can be directly calculated in our ray tracing software by using a specially designed iterative scheme.

There are several effects related to the propagation delays such as just the constant shift of the aberration patterns due to the change of the orientation of the instrument during the propagation time: an image of a star observed at time $t_{\mathrm{obs}}$ is produced by the light rays from the star that hit the primary mirror at time $\sim t_{\mathrm{obs}}-d_{\mathrm{f}} / c$ when the orientation of the mirror differed by $\sim \Omega d_{\mathrm{f}} / c$ from the orientation at $t_{\mathrm{obs}}$. Similar constant shifts will be caused by intermediate mirrors and by the motion of the focal plane during the propagation delay: during the light propagation the focal plane is moving and the photon hits the focal plane at different positions which correspond to different positions on the sky. This can be computed as $\sim \Omega\left(d_{\mathrm{f}}-r\right) / c$ for the one-mirror system depicted in Fig. 1. Note that in the limit when the center of rotation is infinitely far from the instrument (that is, when all parts of the instrument effectively have the same velocity), these constant shifts are fully equivalent to the normal aberration of light. The constant shifts of the aberration patterns, that can be relatively large, lead only to a constant time shift in the orientation parameters of the satellite derived from astrometric observations: the orientation obtained from observations at $t_{\mathrm{obs}}$ is actually the orientation the satellite had some small earlier time interval. This has only slight consequences on the measurements for any existing or planned astrometric projects. However, the propagation delays also lead to a deformation of the aberration patterns that depends on the field angles. These aberration pattern deformations together with the deformations due to the relativistic reflection law can be important as illustrated below. The distortions of the shape of the patterns are caused by different velocities of different parts of both mirrors and slightly different incident angles for each mirror.

For the one-mirror case these effects are illustrated in Fig. 2. The nine patterns in each of the three panels correspond to nine combinations of the field angles with $a_{\mathrm{L}}=-30^{\prime}, 0^{\prime},+30^{\prime}$ (horizontal direction) and $a_{\mathrm{C}}=-30^{\prime}, 0^{\prime},+30^{\prime}$ (vertical direction). For the focal length $d_{\mathrm{f}}=46.67 \mathrm{~m}, 30^{\prime}$ corresponds to about $407 \mathrm{~mm}$ in the focal plane coordinates. The size of the axes in focal plane coordinates is $0.5 \mathrm{~mm} \times 0.5 \mathrm{~mm}$ for all patterns. The aberration patterns in the upper panel are calculated for a non-rotating instrument. In the middle panel the aberration patterns are obtained using the usual reflection law, but the effects of the light propagation delays are taken into account. In the lower panel both the light propagation delays and the relativistic reflection law are used. An extremely high angular velocity $\Omega=5 \times 10^{9}$ " $/ \mathrm{s}$ is used to exaggerate the distortion and make it clearly visible. The three rightmost patterns in both the middle and the lower panels are much larger than all other patterns. These six patterns extend to the left from the edge of the figure by about 3 times the size of the horizontal axis in each pattern. These parts of the patterns are not shown in Fig. 2. The axes for each pattern are centered at the corresponding photocenter. Note that these photocenters are 
Table 1. The shifts of the aberration patterns for the one-mirror optical system rotating at $\Omega=60^{\prime \prime} / \mathrm{s}$ after subtracting the mean value $\delta \bar{a}_{\mathrm{L}}^{\mathrm{d}}+$ $\delta \bar{a}_{\mathrm{L}}^{\mathrm{r}}=18.3834 \mu$ as.

\begin{tabular}{c|rrr|rrr}
\hline \hline & \multicolumn{3}{|c|}{$\delta a_{\mathrm{L}} \times 10^{-3} \mu$ as } & \multicolumn{3}{|c}{$\delta a_{\mathrm{C}} \times 10^{-3} \mu$ as } \\
$a_{\mathrm{C}} \backslash a_{\mathrm{L}}$ & $-30^{\prime}$ & $0^{\prime}$ & $+30^{\prime}$ & $-30^{\prime}$ & $0^{\prime}$ & $+30^{\prime}$ \\
\hline$-30^{\prime}$ & 0.9 & -1.2 & 0.9 & 1.4 & 0.0 & -1.4 \\
$0^{\prime}$ & 0.2 & -1.9 & 0.2 & 0.0 & 0.0 & 0.0 \\
$30^{\prime}$ & 0.9 & -1.2 & 0.9 & -1.4 & 0.0 & 1.4 \\
\hline
\end{tabular}

significantly shifted between the three panels due to the constant propagation time effects discussed above.

Since for the one-mirror instrument the angle of each light ray with respect to the normal to the mirror at each point of the surface is not greater than $30^{\prime}$, the effect of the relativistic reflection law on aberration patterns is very small. At point $\mathrm{P}_{1}$ the velocity vector is perpendicular to the normal to the mirror. Therefore, at this point for any $a_{\mathrm{L}}$ and $a_{\mathrm{C}}$ the relativistic reflection law coincides with the usual one (see Eq. (A.44)). A light ray going through that point will intersect the focal plane at the same point for both the usual and relativistic reflection laws. The light rays of the same grid not going through $\mathrm{P}_{1}$ have different images when using the usual reflection law and the relativistic one.

For realistic $\Omega=60^{\prime \prime} / \mathrm{s}$ the mean shift of the photocenters due to the propagation delays amount to $\delta \bar{a}_{\mathrm{L}}^{\mathrm{d}}=18.3842 \mu$ as. Note that this number can be reproduced with good accuracy by $\Omega\left(2 d_{\mathrm{f}}-r\right) / c=18.3807 \mu$ as as discussed above. The field-angle dependent change of the photocenters is at the level of $0.001 \mu$ as and is shown in Table 1 . The change of the photocenters due to the relativistic reflection law is a shift in the along-scan direction $\delta a_{\mathrm{L}} \approx \delta \bar{a}_{L}^{\mathrm{r}}=-0.0008 \mu$ as and is independent of $a_{\mathrm{L}}$ and $a_{\mathrm{C}}$ at the level of $0.0001 \mu$ as.

\subsection{A two-mirror optical system}

Real optical systems usually have more than one mirror. Often the instruments involve mirrors inclined by about $45^{\circ}$ to the optical axis (i.e., Nasmith focus, beam combiners, beam splitters, etc.). In this case the effects of the relativistic reflection law on the aberration pattern are significantly larger than in the case discussed above. Here we consider an optical system consisting of one parabolic primary mirror and one flat secondary mirror as depicted in Fig. 3. A flat secondary mirror $M_{2}$ has been added to the optical system depicted in Fig. 1. The distance from $\mathrm{P}_{1}$ to center of the flat mirror $\mathrm{P}_{2}$ is $d_{12}$. The whole system is again rigidly rotating with a constant angular velocity $\Omega$ in laboratory coordinates. The flat mirror is inclined at an angle $\theta$ with respect to the optical axis of the primary mirror. The focal plane position depends on the angle $\theta$. The distance from $\mathrm{P}_{1}$ to $\mathrm{P}_{2}$ is $d_{12}=3 \mathrm{~m}$, and the distance from $\mathrm{P}_{1}$ to the rotational axis $\mathrm{O}$ is $r=1.5 \mathrm{~m}$. The distance from $\mathrm{P}_{2}$ to the center $C$ of the focal plane is $d_{\mathrm{f}}-d_{12}=d_{2 f}=43.67 \mathrm{~m}$. The bold line in Fig. 3 representing the optical axis goes from $\mathrm{P}_{1}$ to $\mathrm{P}_{2}$ and then to the focal plane center $C$.

We repeat the ray tracing calculations as described in Sect. 3.1 above with this additional flat mirror. We use three different configurations of the flat mirror with inclination angles $\theta=+45^{\circ}, \theta=0$, and $\theta=-45^{\circ}$. Figure 4 shows the aberration patterns obtained with $\theta=45^{\circ}$ (again for a large angular velocity of $\Omega=5 \times 10^{7 \prime \prime} / \mathrm{s}, 100$ times lower than for Fig. 2, was used in order to make the effects visible). The same

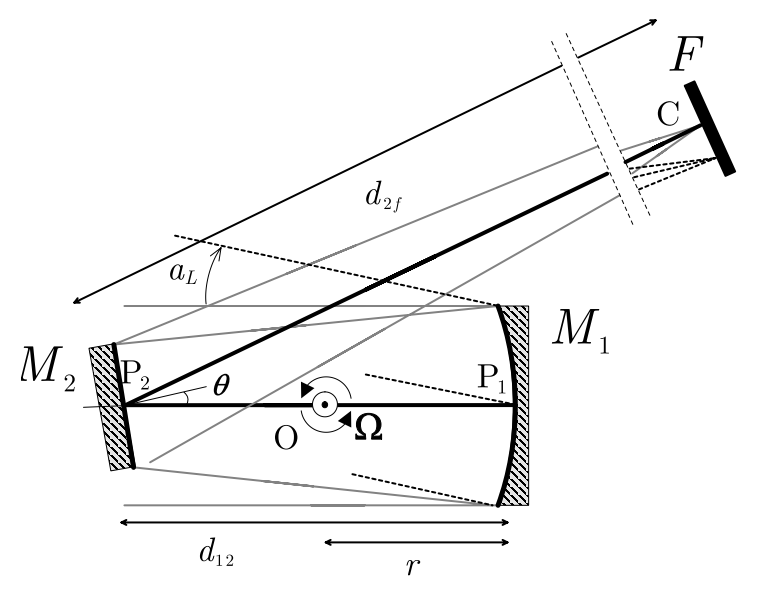

Fig. 3. Two-mirror optical system.

Table 2. The shifts of the aberration patterns for the two-mirror optical system rotating at $\Omega=60^{\prime \prime} / \mathrm{s}$ for three values of $\theta$ after subtracting the specified mean values $\delta \bar{a}_{\mathrm{L}}^{\mathrm{d}}+\delta \bar{a}_{\mathrm{L}}^{\mathrm{r}}$.

\begin{tabular}{|c|c|c|c|c|c|c|}
\hline \multirow{3}{*}{$\begin{array}{c}\theta=-45^{\circ}: \\
a_{\mathrm{C}} \backslash^{a_{\mathrm{L}}}\end{array}$} & \multicolumn{6}{|c|}{ 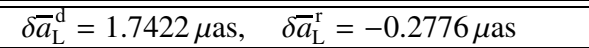 } \\
\hline & \multicolumn{3}{|c|}{$\delta a_{\mathrm{L}} \times 10^{-3} \mu \mathrm{as}$} & \multicolumn{3}{|c|}{$\delta a_{\mathrm{C}} \times 10^{-3} \mu \mathrm{as}$} \\
\hline & $-30^{\prime}$ & $0^{\prime}$ & $+30^{\prime}$ & $-30^{\prime}$ & $0^{\prime}$ & $+30^{\prime}$ \\
\hline$-30^{\prime}$ & 4.6 & 0.0 & -4.5 & 4.8 & 4.9 & 4.9 \\
\hline $0^{\prime}$ & 4.5 & -0.1 & -4.6 & 0.0 & 0.0 & 0.0 \\
\hline $30^{\prime}$ & 4.6 & 0.0 & -4.5 & -4.8 & -4.9 & -4.9 \\
\hline \multirow[t]{2}{*}{$\theta=0^{\circ}:$} & \multicolumn{6}{|c|}{$\delta \bar{a}_{\mathrm{L}}^{\mathrm{d}}=2.0246 \mu \mathrm{as}, \quad \delta \bar{a}_{\mathrm{L}}^{\mathrm{r}}=0.0006 \mu$ as } \\
\hline & \multicolumn{3}{|c|}{$\delta a_{\mathrm{L}} \times 10^{-3} \mu$ as } & \multicolumn{3}{|c|}{$\delta a_{\mathrm{C}} \times 10^{-3} \mu \mathrm{as}$} \\
\hline$a_{\mathrm{C}} \backslash a_{\mathrm{L}}$ & $-30^{\prime}$ & $0^{\prime}$ & $+30^{\prime}$ & $-30^{\prime}$ & $0^{\prime}$ & $+30^{\prime}$ \\
\hline$-30^{\prime}$ & 0.0 & 0.0 & 0.0 & 0.0 & 0.0 & 0.0 \\
\hline $0^{\prime}$ & 0.0 & 0.0 & 0.0 & 0.0 & 0.0 & 0.0 \\
\hline $30^{\prime}$ & 0.0 & 0.0 & 0.0 & 0.0 & 0.0 & 0.0 \\
\hline \multicolumn{7}{|l|}{$\theta=+45^{\circ}:$} \\
\hline & \multicolumn{3}{|c|}{$\delta a_{\mathrm{L}} \times 10^{-3} \mu \mathrm{as}$} & \multicolumn{3}{|c|}{$\delta a_{\mathrm{C}} \times 10^{-3} \mu \mathrm{as}$} \\
\hline$a_{\mathrm{C}} \backslash^{a_{\mathrm{L}}}$ & $-30^{\prime}$ & $0^{\prime}$ & $+30^{\prime}$ & $-30^{\prime}$ & $0^{\prime}$ & $+30^{\prime}$ \\
\hline$-30^{\prime}$ & -4.5 & 0.0 & 4.6 & -4.9 & -4.9 & -4.8 \\
\hline $0^{\prime}$ & -4.6 & -0.1 & 4.5 & 0.0 & 0.0 & 0.0 \\
\hline $30^{\prime}$ & -4.5 & 0.0 & 4.6 & 4.9 & 4.9 & 4.8 \\
\hline
\end{tabular}

9 combinations of $a_{\mathrm{L}}$ and $a_{\mathrm{C}}$, and the same size and centering of the axes are used for each panel as described above for Fig. 2. The upper panel shows the aberration patterns for a non-rotating instrument $(\Omega=0)$. These patterns are identical to those in the left panel of Fig. 1. Clearly, the aberration patterns for the rotating instrument, the middle and the lower panel look differently to Fig. 2. Numerical values of the shifts of the photocenters $\delta a_{\mathrm{L}}$ and $\delta a_{\mathrm{C}}$ for $\Omega=60^{\prime \prime} / \mathrm{s}$ are presented in Table 2 for inclination angles $\theta=45^{\circ}, 0^{\circ},-45^{\circ}$. The mean constant shift $\delta \bar{a}_{\mathrm{L}}^{\mathrm{d}}$ of the patterns due to the light propagation delays and $\delta \bar{a}_{\mathrm{L}}^{\mathrm{r}}$ due to the relativistic reflection law are given at the top of each table. The tables show the part of the total shifts dependent on the field angles. The position-dependent effects in $\delta a_{\mathrm{L}}^{\mathrm{d}}$ and $\delta a_{\mathrm{L}}^{\mathrm{r}}$ have opposite signs and are 2-3 times larger than the total shift $\delta a_{\mathrm{L}}=\delta a_{\mathrm{L}}^{\mathrm{d}}+\delta a_{\mathrm{L}}^{\mathrm{r}}$. On the contrary, the effects in $\delta a_{\mathrm{C}}^{\mathrm{d}}$ and $\delta a_{\mathrm{C}}^{\mathrm{r}}$ are of the same sign and are about 2 times less than in the sum $\delta a_{\mathrm{C}}=\delta a_{\mathrm{C}}^{\mathrm{d}}+\delta a_{\mathrm{C}}^{\mathrm{r}}$. 

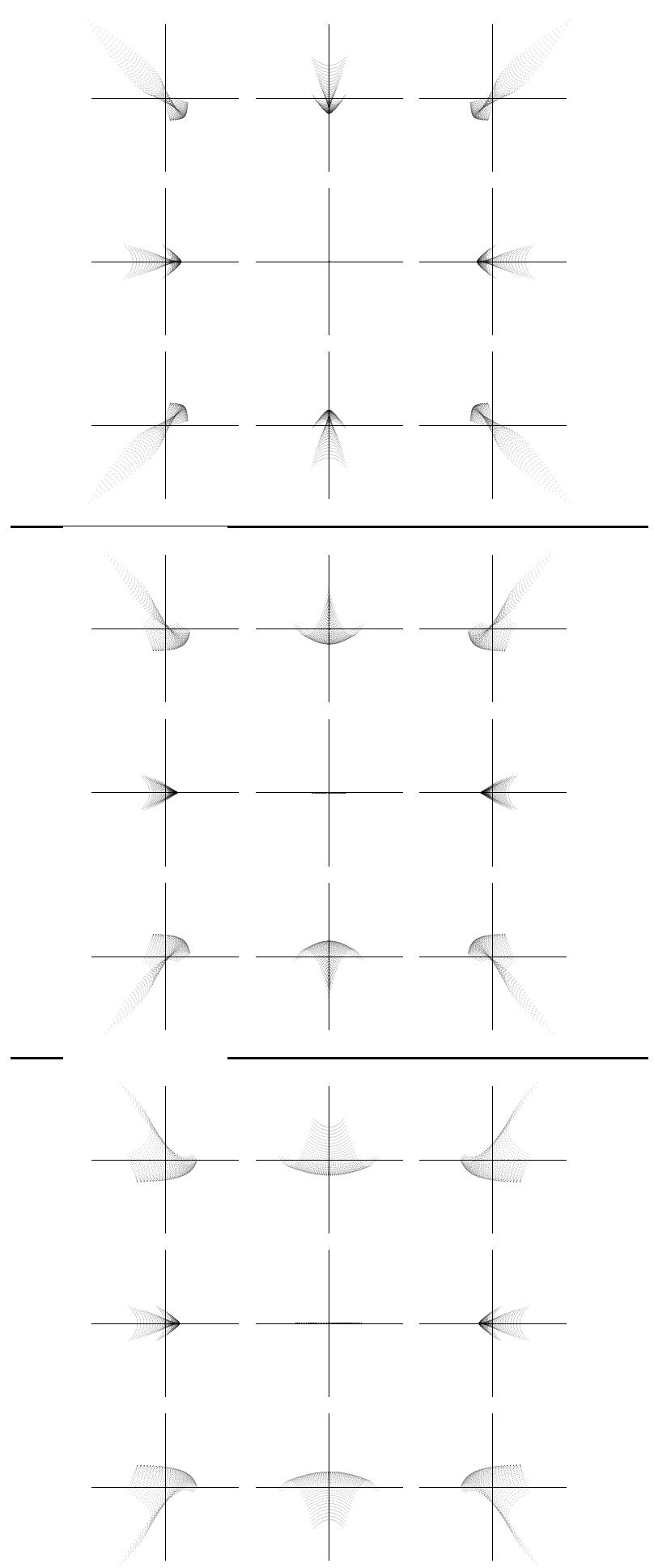

Fig. 4. Aberration patterns for the two-mirror system with $\theta=45^{\circ}$ : non-rotating instrument (upper panel), rotating instrument considering the light propagation delays and using the usual reflection law (middle panel), and rotating instrument considering both the light propagation delays and the relativistic reflection law (lower panel).

As for the one-mirror system, for any value of $\theta$ the shifts due to the light propagation delays exceed the level of $1 \mu$ as and amount to $\delta \bar{a}_{\mathrm{L}}^{\mathrm{d}} \sim 2 \mu \mathrm{as}$. For the two-mirror system $\delta \bar{a}_{\mathrm{L}}^{\mathrm{d}}$ is significantly lower than for the one-mirror system since the effects of the motion of the primary mirror and the motion of the focal plane largely compensate each other if just one intermediate mirror is present.

For $\theta=0$ the shifts due to the relativistic deflection law are again very small as was the case for the one-mirror system. The situation with these shifts is different for $\theta= \pm 45^{\circ}$ where the mean shift $\delta \bar{a}_{\mathrm{L}}^{\mathrm{r}} \sim 0.3 \mu$ as. For $\theta= \pm 45^{\circ}$ all the light rays hit the flat surface at an angle of about $\alpha= \pm 45^{\circ}$ with respect to the normal and the factor $|\sin \alpha|$ appearing in (A.60) is of the order of $1 / \sqrt{2} \approx 0.7$. Each light ray of the grid hits the mirror at a slightly different value of $\alpha$, but the main perturbation due to the relativistic reflection law can be estimated considering the light ray going along the optical axis. Using (A.60) we obtain

$\delta_{2} \simeq 2 \frac{v}{c} \frac{d_{2 \mathrm{f}}}{d_{\mathrm{f}}} \sin ^{2} \theta$,

where $d_{2 \mathrm{f}}$ is again the distance between $\mathrm{P}_{2}$ and the focal plane center as shown in Fig. 3, and $v$ is the velocity of the point of the mirror lying on the optical axis $\left(v=\Omega\left(d_{12}-r\right)\right.$ for the case depicted in Fig. 3). One can check that the mean constant shifts $\delta \bar{a}_{\mathrm{L}}^{\mathrm{r}}$ as shown in Table 2 can be recovered from (1) almost exactly. If more flat (or almost flat) mirrors are added, the expression can be generalized by

$\left|\delta_{i}\right| \simeq\left|2 \frac{v_{i}}{c} \frac{d_{i \mathrm{f}}}{d_{\mathrm{f}}} \sin \theta_{i} \sin \varphi_{i}\right|$.

The index $i$ is used to enumerate the surfaces along the light path, $i=1$ corresponding to the primary mirror. In our case $i=1$ is the parabolic mirror $M_{1}$ and $i=2$ is the flat mirror $M_{2}$. The angle $\varphi_{i}$ is the angle between the velocity and the surface at the intersection of the mirror $M_{i}$ with the optical axis applying the conventions described in Fig. A.1. $\theta_{i}$ is the angle between the optical axis and the normal to the surface at the point of intersection. The quantity $d_{i f}$ is the distance from the center of the focal plane $C$ to the point where the optical axis crosses the $i$ th mirror. As defined above $d_{\mathrm{f}}$ is the focal distance of the optical system.

The presence of the factor $d_{i f} / d_{\mathrm{f}}$ in (1) and (2) can be explained by a small perturbation $\Delta$ of the propagation direction of a light ray by a mirror located at a distance $d_{i f}$ from the focal plane causing a linear shift in the focal plane $d_{i f} \Delta$ which is efficiently interpreted as an angular shift of $d_{i f} / d_{\mathrm{f}} \Delta$. In the more general case when the intermediate reflecting surfaces are not flat, Eq. (2) is no longer valid, but gives a reasonable idea of the magnitude of the effect provided that all reflecting surfaces are not too different from a flat mirror. The cumulative effect of a series of (almost) flat mirrors will not be a direct addition of all $\delta_{i}$ since the relativistic perturbation may occur at different planes. An analytic expression in vector form can be derived for the combined effect, but since the resulting formula is complicated and still a rough approximation it will not be discussed here. Equation (2) also has been checked for some other optical systems involving more reflecting surfaces of different shapes, sizes and velocities. A good agreement with the numbers from numerical ray tracing was obtained in all cases.

\section{Concluding remarks}

We have considered in detail the main relativistic effect on the imaging by a rotating optical system which is produced by the relativistic modification of the reflection law. We have considered two simple optical systems containing one and two mirrors. Although the size of the primary mirror, the focal length and the angular velocity of rotation of both systems were defined to 
agree with the corresponding parameters of Gaia, it is not clear how large these effects will be for the real optical scheme of Gaia. We have seen that the effects are small for the one-mirror system and that they may amount of $0.3 \mu$ as for the two-mirror system. For a real Gaia optical scheme the effect may be much larger because of the presence of several inclined mirrors. The two examples of a rotating optical system considered above do not allow us to predict the relativity-induced photocenter shifts for a real optical system like Gaia. A detailed calculation of the photocenter shifts in principle can be done using the ray tracing software developed for this investigation.

The part of the effect that does not depend on the position in the focal plane can be interpreted as a constant change in the orientation of the satellite (as discussed at the end of the previous Section for propagation delay effects). Moreover, if a satellite (like Gaia) has two optically different telescopes, the difference in the main effects for these two telescopes can be interpreted as a change in the angle between the two instruments.

In this paper we confined ourselves to ray tracing in the geometric optics limit. A more strict way to analyze the imaging by a rotating optical system is to apply wave optics and calculate corresponding intensity patterns (PSF or similar characteristics). The intensity patterns would then allow us to predict the observable shifts of the photocenters more reliably than the aberration patterns used in this paper. Preliminary calculation with a simplified model fosters the hope that at optical wavelengths the differences in the photocenter shifts calculated from ray tracing and from wave optics are negligible. However, the effects of propagation delays due to the rotation of the telescope may play a role. This deserves separate investigation.
Acknowledgements. S.K. and M.S. were partially supported by the BMWi grant 50 QG 0601 awarded by the Deutsche Zentrum für Luft- und Raumfahrt e.V. (DLR). The work of G.A. and J.T. was possible thanks to the grant PNE-2003-04352 awarded by the Ministerio de Ciencia y Tecnologia (SPAIN).

\section{References}

Bienaymé, O., \& Turon, C. 2002, GAIA: A European Space Project, Vol. textbook (Les Ulis: EDP Sciences)

Bolotovskii, B. M., \& Stolyarov, S. N. 1989, Uspekhi Fizicheskikh Nauk, 159(1), 155, in Russian; translated into English as Sov. Phys. Usp., 32, 813, 2003

de Boer, K. S., Gilmore, G. E. H., et al. 2000, GAIA: Composition, Formation and Evolution of the Galaxy, Concept and Technology Study Report (ESA$\mathrm{SCI})$

Einstein, A. 1905, Ann. Physik, 17, 891

Gjurchinovski, A. 2004, Am. J. Phys., 72, 1316

Gouda, N., Tsujimoto, T., Kobayashi, Y., et al. 2002, Ap\&SS, 280, 89

Hickson, P., Bhatia, R., \& Iovino, A. 1995, A\&A, 303, L37

Jackson, J. D. 1975, Classical Electrodynamics (John Wiley and Sons)

Klioner, S. A. 2003, AJ, 125, 1580

Klioner, S. A. 2004, Phys. Rev. D, 69, 124001

Klioner, S. A., \& Voinov, A. V. 1993, Phys. Rev. D, 48, 1451

Mashhoon, B. 2005, Int. J. Mod. Phys. D, 14, 171

Lightman, A. P., Press, W. H., Richard, H. P., \& Teukolsky, S. A. 1975, Problem book in Relativity and Gravitation (Princeton University Press)

Ni, W. T., \& Zimmermann, M. 1978, Phys. Rev. D, 17, 1473

Pauli, W. 1958, Theory of Relativity (Pergamon Press)

Perryman, M. A. C., Lindegren, L., Kovalevsky, J., et al. 1997, A\&A, 323, L49

Perryman, M. A. C., de Boer, K. S., Gilmore, G., et al. 2001, A\&A, 369, 339

Ragazzoni, R., \& Claudi, R. U. 1995, A\&A, 297, L53

Shao, M. 1998, in Astronomical Interferometry, ed. R. D. Reasenberg, Proc. SPIE, 3350, 536

Soffel, M., Klioner, S. A., Petit, G., et al. 2003, AJ, 126, 2687 
G. Anglada-Escudé et al.: Relativistic effects for rotating optical systems, Online Material p 1

\section{Online Material}




\section{Appendix A: Reflection of a light ray by an arbitrarily moving mirror}

\section{A.1. Notation and conventions}

We summarize the most important notation and conventions used throughout the paper:

- $c$ is the velocity of light in vacuum.

- Lowercase Latin indices $a, i, j, \ldots$ take values $1,2,3$ and refer to spatial components of corresponding quantities.

- Index 0 is used for time components.

- Greek indices $\alpha, \mu, v, \ldots$ take values $0,1,2$ and 3 and refer to all space-time components of corresponding quantities.

- The Minkowski metric is denoted by $\eta=$ $\operatorname{diag}(-1,+1,+1,+1)$.

- All Latin indices are lowered and raised by means of the unit matrix $\delta_{i j}=\delta^{i j}=\operatorname{diag}(1,1,1)$, and therefore the position of such indices plays no role: $a^{i}=a_{i}$.

- The symbol $\varepsilon_{i j k}$ is the fully antisymmetric Levi-Civita symbol $\left(\varepsilon_{123}=+1\right)$.

- Repeated indices imply the Einstein summation rule irrespective of their positions (e.g., $a_{i} b_{i}=a_{1} b_{1}+a_{2} b_{2}+a_{3} b_{3}$ ).

- The spatial components of a quantity considered as a 3-vector are set in boldface: $\boldsymbol{a}=a^{i}$.

- The absolute value (Euclidean norm) of a 3-vector $\boldsymbol{a}$ is denoted $|\boldsymbol{a}|$ and is defined by $|\boldsymbol{a}|=\left(a^{1} a^{1}+a^{2} a^{2}+a^{3} a^{3}\right)^{1 / 2}$.

- The scalar product of any two 3-vectors $\boldsymbol{a}$ and $\boldsymbol{b}$ with respect to the Euclidean metric $\delta_{i j}$ is denoted by $\boldsymbol{a} \cdot \boldsymbol{b}$ and is defined by $\boldsymbol{a} \cdot \boldsymbol{b}=\delta_{i j} a^{i} b^{j}=a^{i} b^{i}$.

Two reference systems $\left(t, x^{i}\right)$ and $\left(T, X^{a}\right)$ will be used. All quantities defined in $x^{\mu}=\left(t, x^{i}\right)$ are denoted by small Latin characters with space-time and spatial indices taken from second parts of the Greek and Latin alphabet, respectively $(\mu, v, \ldots, i, j, \ldots)$. All quantities defined in $X^{\alpha}=\left(T, X^{a}\right)$ are denoted by capital Latin characters with space-time and spatial indices taken from first parts of the Greek and Latin alphabet, respectively $(\alpha, \beta, \ldots, a$, $b, \ldots)$.

\section{A.2. Coordinate representation of an arbitrary moving mirror}

Let us consider an inertial reference system of Special Relativity $\left(t, x^{i}\right)$. We define an arbitrary mirror in arbitrary motion by a bundle of particles moving along worldlines

$x_{m}^{\mu}(t ; \xi, \eta)=\left(t, x_{m}^{i}(t ; \xi, \eta)\right)$.

Here $\xi$ and $\eta$ are two parameters "numbering" the particles. These parameters can be though of as some non-degenerate "coordinate system" on the surface of the mirror which is described by $x_{m}^{i}(t ; \xi, \eta)$ for any fixed time $t$. Fixing $\xi$ and $\eta$ we fix a particle on the surface of the mirror and $x_{m}^{i}(t ; \xi, \eta)$ is the worldline of that particle in coordinates $\left(t, x^{i}\right)$. Further, we assume that $x_{m}^{i}(t ; \xi, \eta)$ is differentiable with respect to all its three parameters. This means in particular that the surface of the mirror is assumed to be smooth.

Here we do not pay attention to any physical properties of the mirror as a "physical body" (elasticity, deformations, etc.). We just consider that (A.1) formally defines the position of each point of the mirror at each moment of time. The source of information for $x_{m}^{i}(t ; \xi, \eta)$ for realistic mirrors and the plausibility of these representation of an arbitrarily shaped and arbitrarily moving mirror is discussed in Sect. 2 above.
Starting from (A.1) for any fixed time $t$ at any fixed point of the mirror characterized by some values of $\xi$ and $\eta$ we have two three-dimensional vectors tangent to the surface of the mirror at the considered point as

$$
\begin{aligned}
l^{i} & =\frac{\partial}{\partial \xi} x_{m}^{i}(t ; \xi, \eta), \\
m^{i} & =\frac{\partial}{\partial \eta} x_{m}^{i}(t ; \xi, \eta) .
\end{aligned}
$$

Then a coordinate vector normal to the surface of the mirror at that point can be defined as

$n^{i}=\varepsilon_{i j k} l^{j} m^{k}$.

The order of vectors $l^{i}$ and $m^{i}$ in (A.4) is arbitrary and corresponds to a choice of the sign in the definition of $n^{i}$ (if $n^{i}$ is a normal vector then $-n^{i}$ is also a normal). Not restricting the generality we assume below that (A.4) defines that $n^{i}$ which is directed toward the "working surface" of the mirror, that is for any incoming light ray $\sigma^{i}$ which hits the mirror at the considered point one has $\boldsymbol{\sigma} \cdot \boldsymbol{n}<0$. This normal vector $n^{i}$ has no physical meaning since it is defined in some arbitrary coordinate system $\left(t, x^{i}\right)$. However, it is straightforward to compute $n^{i}$ if $x_{m}^{i}(t ; \xi, \eta)$ is given. Below we show how to relate $n^{i}$ to a physically meaningful normal vector at some point of the mirror as observed by an observer instantaneously co-moving with the considered point of the surface.

The coordinate velocity of any point of the mirror reads

$v_{m}^{i}=\frac{\partial}{\partial t} x_{m}^{i}(t, \xi, \eta)$

\section{A.3. Transforming the mirror surface from one inertial reference system to another}

Let us now define another reference system $\left(T, X^{a}\right)$ moving with constant velocity $v^{i}$ with respect to $\left(t, x^{i}\right)$. The coordinates $\left(T, X^{a}\right)$ and $\left(t, x^{i}\right)$ are related by a Lorentz transformation of the form

$\begin{aligned} c t & =\Lambda_{0}^{0} c T+\Lambda_{a}^{0} X^{a}, \\ x^{i} & =\Lambda_{0}^{i} c T+\Lambda_{a}^{i} X^{a} .\end{aligned}$

The $\Lambda$ matrix coefficients are given by

$\Lambda_{0}^{0}=\gamma$,

$\Lambda_{a}^{0}=k^{a} \gamma$,

$\Lambda_{0}^{i}=k^{i} \gamma$

$\Lambda_{a}^{i}=\delta^{i a}+\frac{\gamma^{2}}{1+\gamma} k^{i} k^{a}$

$\gamma=(1-\boldsymbol{k} \cdot \boldsymbol{k})^{-\frac{1}{2}}$,

$\boldsymbol{k}=\frac{1}{c} \boldsymbol{v}$.

The inverse transformation reads

$c T=\bar{\Lambda}_{0}^{0} c t+\bar{\Lambda}_{i}^{0} x^{i}$,

$X^{a}=\bar{\Lambda}_{0}^{a} c t+\bar{\Lambda}_{i}^{a} x^{i}$.

where $\bar{\Lambda}_{\beta}^{\alpha}$ is equal to $\Lambda_{\beta}^{\alpha}$ with $-\boldsymbol{k}$ substituted for $\boldsymbol{k}$.

In the reference system $\left(T, X^{a}\right)$ the mirror can be also represented in the same form as in Sect. A.2

$X_{m}^{\alpha}(T ; \xi, \eta)=\left(T, X_{m}^{a}(T ; \xi, \eta)\right)$, 
where fixed values for $\xi$ and $\eta$ should correspond to the same surface particle in both coordinate systems. The vectors tangent and normal to the surface read

$$
\begin{aligned}
L^{a} & =\frac{\partial}{\partial \xi} X_{m}^{a}(T ; \xi, \eta), \\
M^{a} & =\frac{\partial}{\partial \eta} X_{m}^{a}(T ; \xi, \eta), \\
N^{a} & =\varepsilon_{a b c} L^{b} M^{c} .
\end{aligned}
$$

Here again, $N^{a}$ is a coordinate normal vector that has no physical meaning. The coordinate velocity of a point of the mirror is given by

$V_{m}^{a}=\frac{\partial}{\partial T} X_{m}^{a}(T ; \xi, \eta)$

Let us now relate the vectors $L^{a}, M^{a}, N^{a}$ and $V_{m}^{a}$ to the corresponding ones in the reference system $\left(t, x^{i}\right)$. We consider the coordinate transformation of the events defined by (A.1) and (A.16)

$$
\begin{aligned}
c T & =\bar{\Lambda}_{0}^{0} c t+\bar{\Lambda}_{i}^{0} x_{m}^{i}(t ; \xi, \eta), \\
X_{m}^{a}(T ; \xi, \eta) & =\bar{\Lambda}_{0}^{a} c t+\bar{\Lambda}_{i}^{a} x_{m}^{i}(t ; \xi, \eta) .
\end{aligned}
$$

The function $X_{m}^{a}(T, \xi, \eta)$ is thus defined by (A.21)-(A.22) implicitly since (A.21) should be inverted to give $t$ as a function of $T, \xi$ and $\eta$ and that $t$ should be substituted into (A.22) to give the explicit dependence of $X_{m}^{a}$ on $T, \xi$ and $\eta$. Clearly, that inversion cannot be done explicitly for any $x_{m}^{i}(t ; \xi, \eta)$. However, the partial derivatives of $X_{m}^{a}(T ; \xi, \eta)$ representing $L^{a}, M^{a}$ and $V_{m}^{a}$ can be calculated as derivatives of an implicit function. Some straightforward algebra gives

$$
\begin{aligned}
V_{m}^{a} & =c \frac{\bar{\Lambda}_{0}^{a}+\bar{\Lambda}_{i}^{a} k_{m}^{i}}{\bar{\Lambda}_{0}^{0}+\bar{\Lambda}_{i}^{0} k_{m}^{i}}, \\
L^{a} & =\bar{S}_{i}^{a} l^{i} \\
M^{a} & =\bar{S}_{i}^{a} m^{i} \\
\bar{S}_{i}^{a} & =\bar{\Lambda}_{i}^{a}-\bar{\Lambda}_{i}^{0} \frac{\bar{\Lambda}_{0}^{a}+\bar{\Lambda}_{j}^{a} k_{m}^{j}}{\bar{\Lambda}_{0}^{0}+\bar{\Lambda}_{j}^{0} k_{m}^{j}},
\end{aligned}
$$

or inverting

$$
\begin{aligned}
v_{m}^{i} & =c \frac{\Lambda_{0}^{i}+\Lambda_{a}^{i} K_{m}^{a}}{\Lambda_{0}^{0}+\Lambda_{a}^{0} K_{m}^{a}}, \\
l^{i} & =S_{a}^{i} L^{a}, \\
m^{i} & =S_{a}^{i} M^{a}, \\
S_{a}^{i} & =\Lambda_{a}^{i}-\Lambda_{a}^{0} \frac{\Lambda_{0}^{i}+\Lambda_{b}^{i} K_{m}^{b}}{\Lambda_{0}^{0}+\Lambda_{b}^{0} K_{m}^{b}},
\end{aligned}
$$

with $k_{m}^{i}=c^{-1} v_{m}^{i}$ and $K_{m}^{a}=c^{-1} V_{m}^{a}$. Equations (A.23) and (A.27) coincide with the law for velocity addition in Special Relativity. One can also check by direct calculation that $S_{a}^{i} \bar{S}_{j}^{a}=\delta_{j}^{i}$ and $\bar{S}_{i}^{a} S_{b}^{i}=\delta_{b}^{a}$.

$$
\text { Using (A.26) and (A.30) }
$$

$$
\begin{aligned}
\bar{S}_{j}^{b} \bar{S}_{k}^{c} \varepsilon_{a b c} & =\frac{1}{\gamma\left(1-\boldsymbol{k} \cdot \boldsymbol{k}_{m}\right)} S_{a}^{i} \varepsilon_{i j k}, \\
S_{b}^{j} S_{c}^{k} \varepsilon_{i j k} & =\gamma\left(1-\boldsymbol{k} \cdot \boldsymbol{k}_{m}\right) \bar{S}_{i}^{a} \varepsilon_{a b c} .
\end{aligned}
$$

Now using these formulas, definitions (A.19) and (A.4) and relations (A.24)-(A.25) and (A.28)-(A.29) one can prove that $N^{a}$ and $n^{i}$ are related as

$$
\begin{aligned}
N^{a} & =\frac{1}{\gamma\left(1-\boldsymbol{k} \cdot \boldsymbol{k}_{m}\right)} S_{a}^{i} n^{i}, \\
n^{i} & =\gamma\left(1-\boldsymbol{k} \cdot \boldsymbol{k}_{m}\right) \bar{S}_{i}^{a} N^{a} .
\end{aligned}
$$

To prove (A.31)-(A.32) we used the identity

$\varepsilon_{a j c} \delta^{k b}+\varepsilon_{k a c} \delta^{j b}+\varepsilon_{j k c} \delta^{a b}=\varepsilon_{a j k} \delta^{b c}$.

\section{A.4. Observable and coordinate normal vectors}

Let us consider an infinitely small element of the mirror which is characterized by infinitely small intervals around some fixed values of $\xi$ and $\eta$. The velocity of the element is $v_{m}^{i}(t ; \xi, \eta)$ in the laboratory reference system $\left(t, x^{i}\right)$. Let us now identify the constant velocity $v^{i}$ of the reference system $\left(T, X^{a}\right)$ relative to $\left(t, x^{i}\right)$ with $v_{m}^{i}(t ; \xi, \eta)$ of the considered point given by $\xi$ and $\eta$ and at some fixed moment of time: $v^{i} \equiv v_{m}^{i}(t ; \xi, \eta)$. Then $\left(T, X^{a}\right)$ is a momentarily co-moving inertial reference system of the considered infinitesimal element of the mirror. The coordinate basis of $\left(T, X^{a}\right)$ gives an orthonormal tetrad of an observer co-moving with the considered element of the mirror. That reference system can be used to describe the results of instantaneous observations made by that observer.

In particular, $N^{a}$ is the observable normal vector which will be used below to formulate the reflection law for the light rays as seen by the co-moving observer. From now on, $N^{a}$ is always used in this sense (that is, we always put $\boldsymbol{k}_{m}=\boldsymbol{k}$ ). Normalizing the vectors one can see that the unit vector $\hat{N}=N /|N|$ normal to the surface as seen by an observer instantaneously co-moving with a particular point of the mirror relates to the normal unit vector $\hat{\boldsymbol{n}}=\boldsymbol{n} /|\boldsymbol{n}|$ seen by an observer at rest relative to $\left(t, x^{i}\right)$ as

$$
\begin{aligned}
& \hat{\boldsymbol{N}}=\frac{1}{\sqrt{1-(\boldsymbol{k} \cdot \hat{\boldsymbol{n}})^{2}}}\left(\hat{\boldsymbol{n}}-(\boldsymbol{k} \cdot \hat{\boldsymbol{n}}) \frac{\gamma}{1+\gamma} \boldsymbol{k}\right), \\
& \hat{\boldsymbol{n}}=\frac{1}{\sqrt{1+\gamma^{2}(\boldsymbol{k} \cdot \hat{N})^{2}}}\left(\hat{N}+(\boldsymbol{k} \cdot \hat{N}) \frac{\gamma^{2}}{1+\gamma} k\right) .
\end{aligned}
$$

This transformation of normal vectors can be derived by the transformation rule of 4 -vectors. Let us again consider a certain surface element in its instantaneously co-moving inertial coordinate system $\left(T, X^{a}\right)$. In that system we consider the 3 -components of the surface normal vector $\hat{N}$ as spatial components of the covariant 4-vector $\hat{N}_{\alpha}=\left(0, \hat{N}^{a}\right)$. A Lorentz transformation of this 4 -vector to coordinates $\left(t, x^{i}\right)$ leads to result (A.37) after normalization.

\section{A.5. Wave vectors in the two inertial reference systems}

In order to consider the light reflection from the mirror we first need to relate the wave vectors of the incoming and outgoing light rays in the two considered coordinate systems. In the reference system $\left(t, x^{i}\right)$ the incoming light ray is characterized by its null wave vector $p^{\mu}\left(\eta_{\mu \nu} p^{\mu} p^{\nu}=0\right)$. The unit light ray direction $\sigma^{i}(\sigma \cdot \sigma=1)$ in that reference system is related to $p^{\mu}$ as $\sigma^{i}=p^{i} / p^{0}$. In the reference system $\left(T, X^{a}\right)$ the null wave vector of the same light ray is $P^{\alpha}$, and the unit light ray direction $\Sigma^{a}=P^{a} / P^{0}(\boldsymbol{\Sigma} \cdot \boldsymbol{\Sigma}=1)$. The frequencies $f$ and $F$ of the light in the corresponding reference systems are linearly proportional to $p^{0}$ and $P^{0}$, respectively. 
The wave vectors $p^{\mu}$ and $P^{\alpha}$ are related by the Lorentz transformation

$P^{\alpha}=\bar{\Lambda}_{\mu}^{\alpha} p^{\mu}$,

which means that the frequencies and unit light ray directions are related as

$$
\begin{aligned}
\Sigma^{a} & =\frac{\bar{\Lambda}_{0}^{a}+\bar{\Lambda}_{i}^{a} \sigma^{i}}{\bar{\Lambda}_{0}^{0}+\bar{\Lambda}_{i}^{0} \sigma^{i},} \\
F & =\left(\vec{\Lambda}_{0}^{0}+\vec{\Lambda}_{i}^{0} \sigma^{i}\right) f .
\end{aligned}
$$

\section{A.6. Reflection as seen by an instantaneously co-moving observer}

For an observer instantaneously co-moving with the element of the mirror where the light ray is reflected the following simple reflection law is valid (in an inertial reference system of Special Relativity for a mirror at rest)

$F^{\prime}=F$,

$\boldsymbol{\Sigma}^{\prime}=\boldsymbol{\Sigma}-2(\hat{\boldsymbol{N}} \cdot \boldsymbol{\Sigma}) \hat{\boldsymbol{N}}$,

where $\hat{N}$ is the observable unit normal vector to the surface of the mirror at the point of reflection as discussed in Sect. A.4 above. The reflection law (A.42) means that the component of $\boldsymbol{\Sigma}$ perpendicular to the surface changes its sign. This automatically guarantees that the angle of incidence is equal to the angle of reflection and that the incoming ray $\boldsymbol{\Sigma}$, the reflected ray $\boldsymbol{\Sigma}^{\prime}$ and the normal $\hat{N}$ are coplanar. The same Eqs. (A.41) and (A.42) are valid for, respectively, the time and space components of wave vectors before and after reflection.

We consider this reflection law as given, but is well known the method to derive it from Maxwell equations for electromagnetic field for a mirror at rest (Jackson 1975). In the instantaneously co-moving reference system $\left(T, X^{a}\right)$ the coordinate velocity of the reflecting point vanishes but its acceleration may differ from zero. However, the acceleration cannot affect the instantaneous process of reflection considering of the equivalence principle as long as the conditions for geometrical optics are satisfied, i.e., as long as the amplitude, polarization and wave vector of an electromagnetic wave do not change significantly over a distance determined by the wavelength $\lambda$. This implies that the acceleration $a_{m}$ of the mirror should satisfy a constraint of the form $a_{m} \ll c^{2} / \lambda$ (see, Mashhoon (2005) for a detailed discussion of accelerated observers in special relativity).

\section{A.7. Reflection as seen by a laboratory observer}

Now combining the reflection law in reference system $\left(T, X^{a}\right)$ with the transformations discussed in Sects. A.3-A.5 one gets the reflection law as seen in reference system $\left(t, x^{i}\right)$ where the mirror is arbitrarily moving

$$
\begin{aligned}
f^{\prime} & =f \frac{1+(\boldsymbol{k} \cdot \hat{\boldsymbol{n}})[\hat{\boldsymbol{n}} \cdot(\boldsymbol{k}-2 \sigma)]}{1-(\boldsymbol{k} \cdot \hat{\boldsymbol{n}})^{2}}, \\
\sigma^{\prime} & =\frac{\left(1-(\boldsymbol{k} \cdot \hat{\boldsymbol{n}})^{2}\right) \sigma+2(\boldsymbol{k} \cdot \hat{\boldsymbol{n}}-\sigma \cdot \hat{\boldsymbol{n}}) \hat{\boldsymbol{n}}}{1+(\boldsymbol{k} \cdot \hat{\boldsymbol{n}})^{2}-2(\boldsymbol{k} \cdot \hat{\boldsymbol{n}})(\sigma \cdot \hat{\boldsymbol{n}})} .
\end{aligned}
$$

Here, $f^{\prime}$ and $\sigma^{\prime}$ are the frequency and the unit direction of the reflected light ray in the reference system $\left(t, x^{i}\right)$. These expressions are valid at each point of the mirror surface in arbitrary motion. Let us remind that $\boldsymbol{k}=\boldsymbol{v}_{m} / c$, where $\boldsymbol{v}_{m}$ is the coordinate velocity of the reflecting point of the mirror at the moment of reflection. Velocity $\boldsymbol{v}_{m}$ can be computed from any mathematical representation of the mirror surface (for example, from (A.5)).

This can be used to derive the 4-momentum or 4-velocity of a particle $p^{\prime \mu}$ after a completely elastic collision with a surface of infinite mass:

$$
\begin{aligned}
& p^{\prime 0}=p^{0}-2 \boldsymbol{k} \cdot \hat{\boldsymbol{n}}\left(\frac{\boldsymbol{p} \cdot \hat{\boldsymbol{n}}-\boldsymbol{k} \cdot \hat{\boldsymbol{n}} p^{0}}{1-(\boldsymbol{k} \cdot \hat{\boldsymbol{n}})^{2}}\right), \\
& p^{\prime i}=p^{i}-2 \hat{n}^{i}\left(\frac{\boldsymbol{p} \cdot \hat{\boldsymbol{n}}-\boldsymbol{k} \cdot \hat{\boldsymbol{n}} p^{0}}{1-(\boldsymbol{k} \cdot \hat{\boldsymbol{n}})^{2}}\right),
\end{aligned}
$$

where $p^{\mu}$ is wave vector of the particle before the collision. Recalling the relations between wave vectors and frequencies and directions for a photon we see that Eqs. (A.45)-(A.46) are equivalent to (A.43)-(A.44).

Let us note two important properties of (A.43)-(A.44), also applicable to (A.45)-(A.46):

1. In the reference system $\left(t, x^{i}\right)$ the reflected direction $\sigma^{\prime}$ also lies in the plane defined by the incoming ray $\sigma$ and the normal vector $\hat{\boldsymbol{n}}$.

2. The reflected ray is only affected by the projection of the velocity $\boldsymbol{v}_{m}$ on the vector $\hat{\boldsymbol{n}}$.

The latter property implies that the relation between $\sigma^{\prime}$ and $\sigma$ coincides with the usual reflection law (A.42) if the velocity $\boldsymbol{v}_{m}$ is perpendicular to $\hat{\boldsymbol{n}}$. This case is relevant for liquid (rotating) mirrors and was discussed by Lightman et al. (1975, problem 1.19), Ragazzoni \& Claudi (1995) and Hickson et al. (1995). Our result (no relativistic effects on reflection law in that case) coincides with that of Lightman et al. (1975) and Hickson et al. (1995).

Multiplying both sides of (A.44) by $\hat{\boldsymbol{n}}$ and using the following definitions for the angles between vectors (see Fig. A.1)

$$
\begin{aligned}
-\boldsymbol{\sigma} \cdot \hat{\boldsymbol{n}} & =\cos \alpha, \\
\boldsymbol{\sigma}^{\prime} \cdot \hat{\boldsymbol{n}} & =\cos \alpha^{\prime}, \\
\boldsymbol{k} \cdot \hat{\boldsymbol{n}} & =k \cos \left(\varphi-\frac{\pi}{2}\right)=k \sin \varphi,
\end{aligned}
$$

( $\left.k=|\boldsymbol{k}|=\left|\boldsymbol{v}_{m}\right| / c\right)$ one obtains a relation between the angle of incidence $\alpha$ and angle of reflection $\alpha^{\prime}$

$$
\begin{aligned}
f^{\prime} & =f \frac{1+2 k \sin \varphi \cos \alpha+k^{2} \sin ^{2} \varphi}{1-k^{2} \sin ^{2} \varphi} \\
\cos \alpha^{\prime} & =\frac{2 k \sin \varphi+\left(1+k^{2} \sin ^{2} \varphi\right) \cos \alpha}{1+k^{2} \sin ^{2} \varphi+2 k \sin \varphi \cos \alpha}
\end{aligned}
$$

The latter equation can be also re-written into an equation relating $\sin \alpha$ and $\sin \alpha^{\prime}$ :

$\sin \alpha^{\prime}=\sin \alpha \frac{1-k^{2} \sin ^{2} \varphi}{1+2 k \sin \varphi \cos \alpha+k^{2} \sin ^{2} \varphi}$

Comparing (A.50) and (A.52) one can see that $f \sin \alpha=$ $f^{\prime} \sin \alpha^{\prime}$.

Angles $\alpha, \alpha^{\prime}$ and $\varphi$ are illustrated in Fig. A.1. The angle $\alpha$ lies between 0 and $\pi / 2$ (since we always consider that the incoming light ray comes to the mirror from one particular side of the tangent plane to the mirror's surface at the point of reflection). For the same reason we have $0 \leq \alpha^{\prime} \leq \pi / 2$. Angle $\varphi$ lies between $-\pi / 2$ and $\pi / 2$. It is negative if the angle between $\boldsymbol{k}$ and $\hat{\boldsymbol{n}}$ is greater than $\pi / 2$ and positive otherwise. 


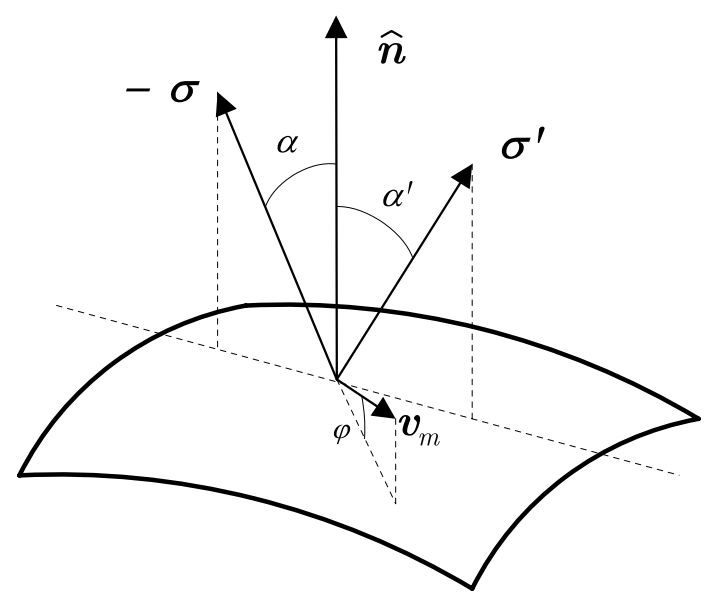

Fig. A.1. Vectors and angles at the point of reflection.

Additionaly, our central results have been derived (A.50)-(A.51) directly from Maxwell's equations by a principle of phase matching: the phase of the incoming wave should agree with the phase of the outgoing one (e.g., Jackson (1975), Sect. 7.3). This generalizes the work of Bolotovskii \& Stolyarov (1989) for a flat mirror moving with constant velocity. For an accelerated mirror such a treatment, however, is meaningful only as long as the conditions for geometrical optics are satisfied.

\section{A.8. Particular case of a flat mirror moving with a constant velocity}

As a particular example let us apply the developed scheme to a flat mirror moving at constant velocity in reference frame $\left(t, x^{i}\right)$. The mathematical expression for that is a worldline Eq. (A.1) of the form

$\boldsymbol{x}_{m}(t, \xi, \eta)=\boldsymbol{x}_{m 0}+\boldsymbol{l} \xi+\boldsymbol{m} \eta+\boldsymbol{v}_{m} t$,

where $\boldsymbol{l}, \boldsymbol{m}, \boldsymbol{v}_{m}$ and $\boldsymbol{x}_{m 0}$ are constant vectors defining position, velocity and orientation of the mirror. In coordinates $\left(T, X^{a}\right)$ one gets

$\boldsymbol{X}_{m}(T, \xi, \eta)=\boldsymbol{X}_{m 0}+\boldsymbol{L} \xi+\boldsymbol{M} \eta+\boldsymbol{V}_{m} T$,

where vectors $\boldsymbol{V}_{m}, \boldsymbol{L}$ and $\boldsymbol{M}$ are related to $\boldsymbol{v}_{m}, \boldsymbol{l}$ and $\boldsymbol{m}$ by (A.23), (A.24) and (A.25), respectively, and $X_{m 0}^{a}=\bar{S}_{i}^{a} x_{m 0}^{i}$. Equation (A.54) implies that a flat surface remains flat in any inertial reference system.
Since for a flat mirror $\boldsymbol{l}$ and $\boldsymbol{m}$ are constants, the unit normal vector $\hat{\boldsymbol{n}}$ is also a constant. Since $\boldsymbol{v}_{m}$ is also time-independent the same reflection law described by (A.44) or (A.51) is valid for any point on the mirror and at any moment of time. (A.51) coincides with the results of Gjurchinovski (2004). Our framework is more general than that of Gjurchinovski (2004) since we do not assume the vectors $\boldsymbol{\sigma}, \hat{\boldsymbol{n}}, \boldsymbol{k}$ and $\boldsymbol{\sigma}^{\prime}$ to be coplanar, and our derivation is valid for an arbitrary mirror in arbitrary motion.

Note that the central result of Gjurchinovski (2004) coincides with the formula derived by Einstein (1905) in the particular case of a flat mirror moving with constant velocity directed perpendicular to the surface when $\sin \varphi=1$ (see also Lightman et al. (1975, problem 1.18)). Bolotovskii \& Stolyarov (1989) have derived the same relation as Einstein (1905) by solving Maxwell field equations directly in the coordinates where the mirror is moving.

\section{A.9. Low velocity limit}

It is useful to derive the first-order expansion of (A.43)-(A.51) in powers of $v_{m} / c$ since in practice the velocity of the mirror will be small compared to the light velocity. One gets

$$
\begin{aligned}
f^{\prime}= & f\left(1-2(\sigma \hat{\boldsymbol{n}})(\boldsymbol{k} \cdot \hat{\boldsymbol{n}})+O\left(c^{-2}\right)\right) \\
\sigma^{\prime}= & \sigma-2(\sigma \cdot \hat{\boldsymbol{n}}) \hat{\boldsymbol{n}} \\
& +2(\boldsymbol{k} \cdot \hat{\boldsymbol{n}})\left[\left(1-2(\sigma \cdot \hat{\boldsymbol{n}})^{2}\right) \hat{\boldsymbol{n}}+(\sigma \cdot \hat{\boldsymbol{n}}) \boldsymbol{\sigma}\right]+O\left(c^{-2}\right)
\end{aligned}
$$

or

$$
\begin{aligned}
f^{\prime} & =f\left(1+2 k \sin \varphi \cos \alpha+O\left(c^{-2}\right)\right), \\
\cos \alpha^{\prime} & =\cos \alpha+2 k \sin \varphi \sin ^{2} \alpha+O\left(c^{-2}\right), \\
\sin \alpha^{\prime} & =\sin \alpha-k \sin \varphi \sin 2 \alpha+O\left(c^{-2}\right) .
\end{aligned}
$$

The first two terms in the right-hand side of (A.56) represent just the usual reflection law and the rest contains the largest relativistic effects. Equation (A.58) shows that

$\alpha^{\prime}-\alpha=-2 k \sin \varphi \sin \alpha+O\left(c^{-2}\right)$.

This expression can be used to estimate the difference $\alpha^{\prime}-\alpha$ for many realistic situations. 